\title{
Development of constitutive equation of filled silicone architectured membrane
}

\author{
M. Rebouah \& G. Chagnon \& D. Favier \\ Université de Grenoble/CNRS, Lab 3SR, Grenoble, France
}

\begin{abstract}
Architectured membranes can be developed in order to mimic living tissues. The main point is to generate anisotropic membranes that can endure large deformations. In this way, crenellated membranes are elaborated with a filled silicone rubber. The aim of this work is to develop a constitutive equation which describes the mechanical behavior of such architectured materials. Membranes with different crenel orientations are elaborated and tested.

The architectured membranes endure the same phenomena as the bulk silicone, they are made with, i.e. principally the Mullins effect for this filled silicone. This phenomenon must be taken account in the modeling. An equivalent constitutive equation is built for the architectured membrane by taking into account the mechanical behavior of the silicone and the geometrical parameters of the crenelated membrane. First, a constitutive equation is chosen to describe the core of the membrane. Second, this equation is adapted to the behavior of the crenels and third a coupling term describing the interactions between the crenels and the membrane is developed. Finally, the model is validated on experimental data.
\end{abstract}

\section{INTRODUCTION}

Architectured materials take a place more and more important in many applications due to their specific properties including mechanical properties (Bouaziz et al., 2008). It exists several types of architectured materials, for instance some materials are architectured due to their microstructure (grain size, polymer chain design, state of crystallization), for metals this strategy operates at scales between $1 \mathrm{~nm}$ and $10 \mu \mathrm{m}$. In other way architectured materials are also composed of the association of several materials (hybrid material) and of geometrical arrangements. This architectured materials allow to build multifonctionnary material (Brechet and Embury, 2013; Ashby, 2013; Bouaziz, 2013). For instance they can be used in many medical applications by means of their ability to exhibit anisotropic behavior. Natural material and particularly soft tissues are architectured materials (Dunlop and Fratzl, 2013).

It is proposed in this paper to study an architectured membrane of filled silicone. Meunier (2011) developed a model and experimental data of an architectured membrane, nevertheless the study is limited to an unfilled silicone rubber Meunier et al. (2009), this rubber is perfectly hyperelastic but suffers from bad fracture properties which makes it difficult to use in real applications.
In this paper it is chosen to use a filled silicone rubber. Due to the fillers this material presents good fracture properties nevertheless it presents too non linear phenomena.

The Mullins effect is the prevailing phenomenon into the used material Machado et al. (2012a), thus the other non linear phenomena as the hysteresis or the relaxation are neglected. A model has already been developed to describe the behavior of this filled rubber silicone for membrane Rebouah et al. (2013).

The aim of this paper is to study the behavior and to build an equivalent model for a filled silicone architectured membrane. The architectured membrane is composed of a flat membrane and crenels on the upper and lower surfaces of this membrane. As described by Meunier (2011) the orientation of the crenels permits to control the anisotropy.

The paper is constructed as follows. In Section 2, the experimental set up is described and the experimental results are presented. In Section 3, a theorical study presents the constitutive equation developed to analyze the behavior of the architectured membrane and a comparison with the experimental results is done. Finally, in Section 4 some conclusions are presented. 


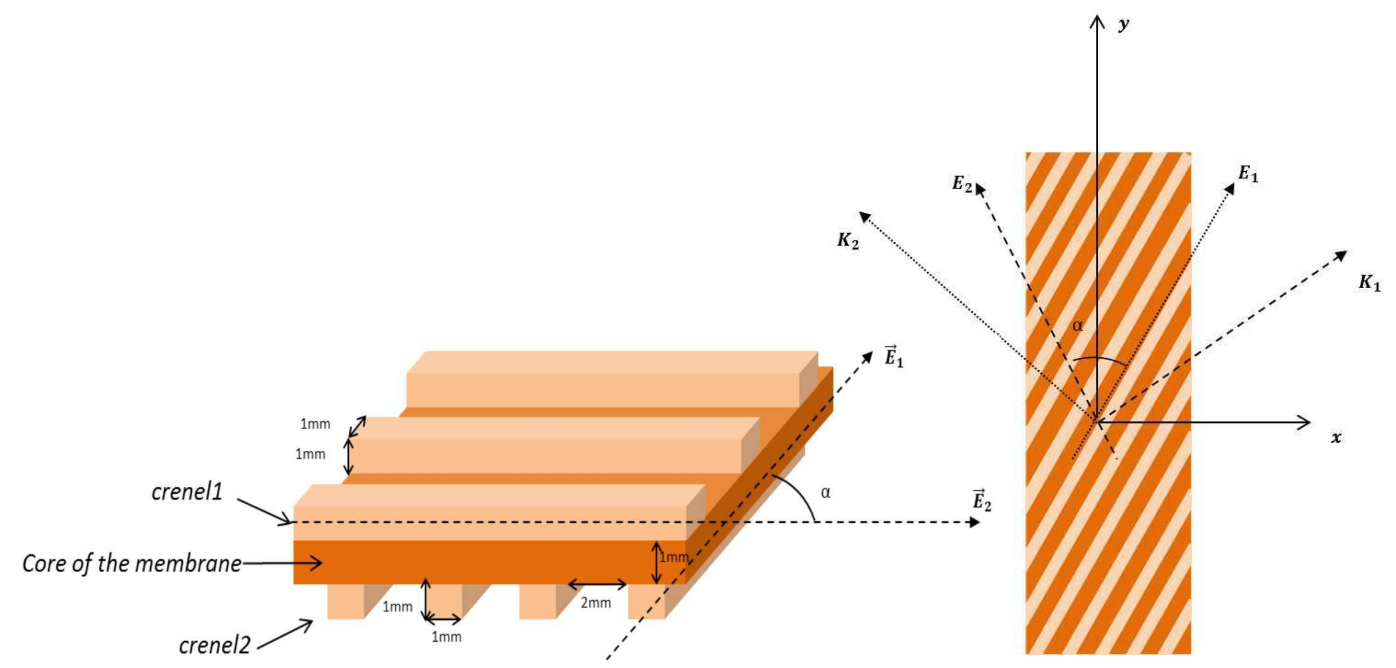

Figure 1: Geometry of the architectured membrane

\section{EXPERIMENTAL STUDY}

Circular crenelated membranes of filled silicone were molded. For each plate, the only parameter that changes is the relative orientation $\alpha$ of the crenels on the upper and lower faces. Five architectured membranes with $\alpha=0^{\circ}, 45^{\circ}, 90^{\circ}, 135^{\circ}$ and $180^{\circ}$ are manufactured and tensile rectangular specimens of $L=60$ $\mathrm{mm}$ long, $\mathrm{l}=21 \mathrm{~mm}$ width and $\mathrm{e}=1 \mathrm{~mm}$ thick for the core of the membrane were cut from the circular plate samples along the direction of traction $\frac{\alpha}{2}$. An illustration is presented in Fig1.

For each architectured membrane rectangular sample, a cyclic tensile test was processed. The samples are loading up to three different but fixed stretches of $\lambda=1.5$, then $\lambda=2$. after unloading, then $\lambda=2.5$ after unloading and finally a reloading to $\lambda=2.5$. The measurements are made by means of a $100 \mathrm{~N}$ sensor which is synchronized to a three-dimensional image correlation (3D-DIC) system to evaluate the strain fields at any point of the surfaces. The specimens present a periodic structure, but boundary conditions can influence the behavior. Thus, a Representative Elementary Volume (REV) is defined in the middle of the sample. Due to the crenels orientations, the section of the specimens is varying with the angle, thus it is proposed to analyze the results using the tension $T$

$T=\frac{F}{w}$

where $F$ represents the force and $w$ the actual width of the sample.

All experimental data are presented in Fig.2. The Fig.2(a) represents the evolution of the transverse elongation during the tensile test. The continuum line represents the theorical transverse elongation for a tensile test and the dashes line represents the transverse elongation obtained for different orientations $\alpha$. For orientations of $\alpha=0^{\circ}, 45^{\circ}, 90^{\circ}$ the transversal elongation is close to the isotropic material $\left(\lambda_{2}=\frac{1}{\sqrt{\lambda_{1}}}\right.$ where $\lambda_{2}$ is the transversal elongation and $\lambda_{1}$ the maximal principal elongation), but for $\alpha=135^{\circ}, 180^{\circ}$ the transversal elongation does not match to the theory of uniaxial tensile test for isotropic material. The Fig.2(b) illustrates the evolution of the tension in function of the tensile elongation. It is observed that the orientation between the crenels $\alpha$, influence the behavior of the material. The lower orientation requires the higher tension to deform the architectured membrane, at the opposite the higher value of $\alpha$ induce a lower tension. If $\alpha$ is superior to $135^{\circ}$ the tension necessary to deform the material decreases very slowly. This results are similar to Meunier (2011) and proves the existence of an induced anisotropy by the architectured membrane.

\section{EQUIVALENT MODEL CONSTITUTIVE EQUATIONS}

RTV3428 is a filled silicone rubber which presents several non linear effects like hysteresis, relaxation (Machado et al., 2010; Rey et al., 2013) and induced anisotropy (Machado et al., 2012b). As illustrated by Rey et al. (2013) the Mullins effect is the prevailing non linear phenomenon so this study focuses on the introduction of this phenomenon into the constitutive equation of the architectured membrane. The other phenomena are not taken into account in a first approach. Rubber like materials are described by means of an hyperelastic strain energy function that can evolve according to the Mullins effect. It is proposed to decompose the strain energy function $\mathcal{W}$ as the sum of the strain energies of the core of the membrane $\mathcal{W}$ (core-membrane) and of each crenel $\mathcal{W}$ (crenel-1), $\mathcal{W}$ (crenel-2), a last term taking into account the coupling between the two directions in the membrane $\mathcal{W}$ (coupling) is added.

$\mathcal{W}=\mathcal{W}($ core-membrane $)+\mathcal{W}($ crenel- 1$)$

$$
+\mathcal{W}(\text { crenel- } 2)+\mathcal{W}(\text { coupling })
$$



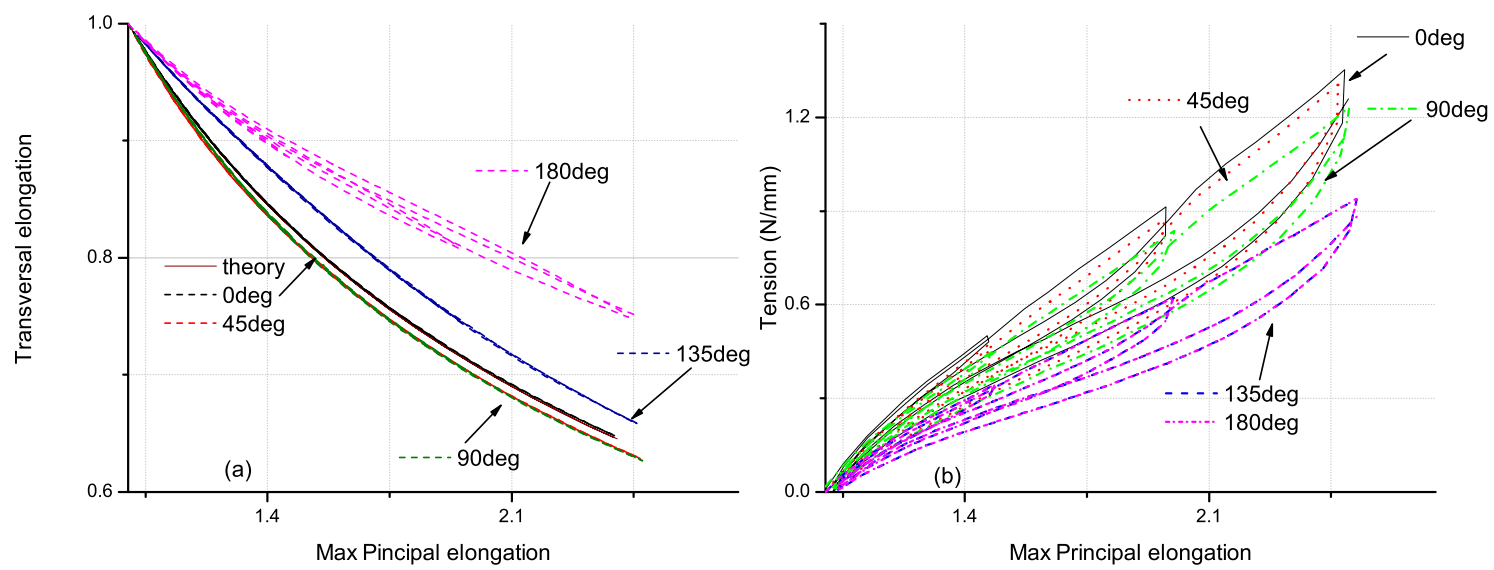

Figure 2: Evolution of the transverse elongation (a) and tension (b) of the architectured membrane for $\alpha=0^{\circ}, 45^{\circ}, 90^{\circ}, 135^{\circ}, 180^{\circ}$

\subsection{Core of the membrane}

The model proposed by Rebouah et al. (2013) which take into account the induced anisotropy by a stress softening is used, it is written as : $\mathcal{W}($ core-membrane $)=\mathcal{W}_{c c}\left(I_{1}, I_{2}\right)+\sum_{i=1}^{42} \omega^{(i)} \mathcal{F}^{(i)} \mathcal{W}_{c f}^{(i)}\left(I_{4}^{(i)}\right)_{\text {sponding to its direction } I_{4 c}^{(i)}=\operatorname{tr}\left(\boldsymbol{C} .\left(\mathbf{E}^{(i)} \otimes \mathbf{E}^{(i)}\right) \text {, i.e: }\right.}^{\text {that implies that the behavior of each crenel can be }}$

The Mooney (1940) strain energy function is chosen for $\mathcal{W}_{c c}$ where $I_{1}, I_{2}$ are the first and second strain invariants of the right Cauchy-Green strain tensor $\mathbf{C}$ and $\omega^{(i)}$ represents the weight of each direction, $\mathcal{F}^{(i)}$ is the Mullins effect evolution function and $W_{c f}^{(i)}$ is the strain energy of each direction. A different form from Rebouah et al. (2013) is used

$\mathcal{W}_{c f}^{(i)}=\frac{K}{2} I_{4}^{(i)}\left(\ln I_{4}^{(i)}-1\right)$

Where $K$ is a material parameter. The evolution function which depends on the first and fourth invariants with only one material parameter $\eta$ is considered :

$\mathcal{F}^{(i)}=1-\eta \sqrt{\frac{I_{1 \max }-I_{1}}{I_{1 \max }-3}}\left(\frac{I_{4 \max }^{(i)}-I_{4}^{(i)}}{I_{4 \max }^{(i)}-1}\right)\left(\frac{I_{4 \max }^{(i)}}{I_{4 \max }}\right)^{4}$

\subsection{The crenels}

The crenels are processed with the same material as the core of the membrane, so the mechanical characteristics are the same. During the deformation, it can be considered that crenels are only submitted to tension-compression, it is estimated that there is no flexion in the crenels and that they can rotate, so they are modeled as bars. To determine the stress of each

$$
\mathcal{W}(\text { crenel-i })=\mathcal{W}\left(I_{4}^{(i)}\right)
$$

crenel, it is proposed to re-writte the Eq.(3) in term of unidirectional components to represent the uniaxial tension endured by the crenels. That means that the second term of the anisotropic part of the strain density energy (Eq.(3)) should be replace by a function of the elongation in the direction $\left(\mathbf{E}_{(i)}\right)$ of the crenel,

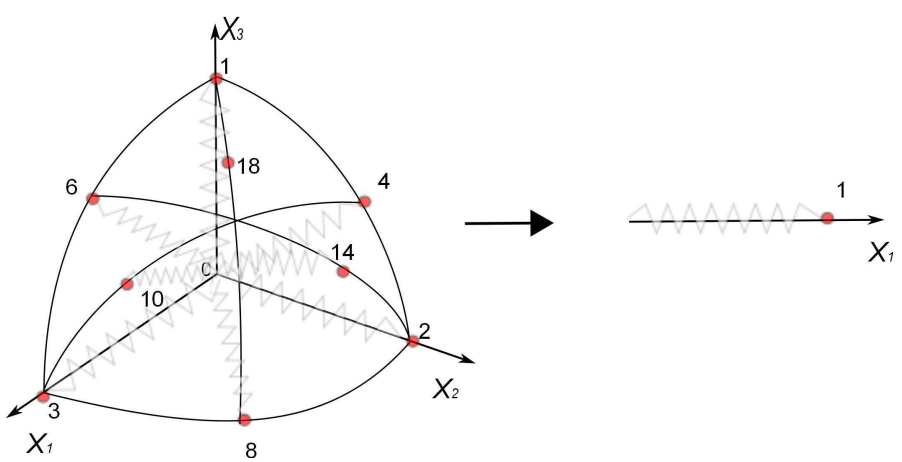

Figure 3: Representation of a eighth the 42 directions of Bazant and Oh (1986) of reimplacement of the 42 directions by only one.

Let us define $\mathbf{B}_{\mathbf{c}}{ }^{(i)}$ as the left Cauchy-Green deformation tensor for each crenel. Since the crenel is only in tension-compression, $\mathbf{B}_{\mathbf{c}}{ }^{(i)}$ is defined in the basis of each crenel $R^{(i)}\left(\mathbf{E}_{(i)}, y^{(i)}, z^{(i)}\right)$ where $y^{(i)}$ and $z^{(i)}$ are vectors which permit to obtain an orthogonal basis.

$\mathbf{B}_{c}^{(i)}=\left(\begin{array}{ccc}\lambda_{\mathbf{E}_{(i)}}^{2} & 0 & 0 \\ 0 & \frac{1}{\lambda_{\mathbf{E}(i)}} & 0 \\ 0 & 0 & \frac{1}{\lambda_{\mathbf{E}(i)}}\end{array}\right)$

Where $\lambda_{\bar{E}_{(i)}}$ is the elongation of the crenel and depend of the fourth invariant $I_{4 c}^{(i)}=\lambda_{E(i)}^{2}$.. In the same way the first $\left(I_{1 c}^{(i)}\right)$, second $\left(I_{2 c}^{(i)}\right)$ and fourth $\left(I_{4 c}^{(i)}\right)$ invariant 
are defined in the basis of each crenel $R^{(i)}$

By means of the Bazant theory it is known that the description of the behavior in only 1 direction modifies too the weight $\omega_{c}^{(i)}$ which has to be recalculated By considering, the projection of the 42 directions, it is possible to replace these directions by only one direction affected with a new weight $\tilde{\omega}=0.21$.

The evolution function was described as the product of three terms (cf Eq.(5)). The third term is useless with only one direction. According to the definition of the fourth invariant, the first and the second term are dependent since $I_{4 c}$ exists only for one direction. It is chosen to express the evolution function only with the fourth invariant, the evolution function is adapted as:

$\mathcal{F}_{c}^{(i)}=1-\eta \chi\left(\frac{I_{4 c \max }^{(i)}-I_{4 c}^{(i)}}{I_{4 c \max }^{(i)}-1}\right)^{2}$

Where $\chi$ represents the intensity of the evolution function for one direction instead of 42 . The intensity $\chi$ of the crenels evolution function must be of 0.5 to limit the rupture of slope.

Finally, the strain energy density of the crenel is directly expressed as:

$$
\mathcal{W}\left(\text { crenel }_{i}\right)=\mathcal{W}_{c c}\left(I_{4 c}^{(i)}\right)+\tilde{\omega} \mathcal{F}_{c}^{(i)} \mathcal{W}_{c f c}^{(i)}\left(I_{4 c}^{(i)}\right)
$$

Nevertheless the comportment described previously is in fact for a membrane behavior, not for crenels, so due to the geometry of the architectured membrane a new quantity is introduced to evaluate the proportion of crenels on the upper and lower faces of the flat membrane $\beta$. According to the structure of the architectured membrane (Fig.1) we deduce $\beta=\frac{1}{3}$

\subsection{Strain energy of coupling}

It is proposed to develop a term to take into account the coupling effects between the crenels and the core of the membrane. The strain energy of the coupling term takes into account the shear induced by the crenels on the external faces of the core of the membrane, i.e. along the two directions $\bar{E}_{1}$ and $\bar{E}_{2}$. Different coupling terms were proposed in literature. Reese et al. (2001) developed a strain energy coupling between orthotropic directions composed by the product of terms isotropic and anisotropic or just anisotropic to control easily the influence of each term. Natali et al. (2009) developed a term of coupling for living tissues by using the eighth and ninth invariant. Göktepe et al. (2010) and Nerurkar et al. (2011) define also a constitutive equations by using the eight invariant to model living tissue as heart or annulus fibrosus of the intervertebral disc. It is proposed here to developed a coupling term by using the eight and ninth invariant too

$\mathcal{W}($ coupling $)=\mathcal{W}\left(I_{8}, I_{9}\right)$
Where $I_{8}$ allows to take into account the shear dependent of $\alpha$ and $I_{9}$ to norm the strain energy density, by adaptation of Spencer (1971):

$$
\begin{aligned}
& I_{8}=\frac{1}{2}\left(\mathbf{C}: \mathbf{E}^{(1)} \otimes \mathbf{E}^{(2)}+\mathbf{C}: \mathbf{E}^{(2)} \otimes \mathbf{E}^{(1)}\right) \\
& I_{9}=\mathbf{E}^{(1)} \cdot \mathbf{E}^{(2)}
\end{aligned}
$$

The strain energy density proposed is:

$$
\mathcal{W}(\text { coupling })=\int C_{8}(\alpha) \ln \left(1+I_{8}-I_{9}\right) d I_{8}
$$

Where $C_{8}(\alpha)$ represents a parameter dependent on the orientation $\alpha$ between the crenels. According to the theory of the hyperelasticity the stress term of coupling is easily deduced. A quadratic function is used to describe the evolution of the coupling term with the angle

$C_{8}(\alpha)=a \alpha^{2}$

\subsection{Comparisons with experimental data}

The total strain energy were implemented in a Finite Element code, the simulation results of cyclic tensile tests were compared to the experimental data as illustrated in Fig.4. A good match with the experimental results for each orientation of the crenels is observed. For each mechanical parameters of the filled silicone the following values were used $\left(C_{1}=0.065\right.$, $\left.C_{2}=0.045, \eta=9, K=0.41\right)$, this values are fitted only on a membrane of filled silicone. The only parameter fitted with the equivalent model is the parameter $a$ for the quadratic function that describe the evolution of the coupling term $(a=0.0446)$.

\section{CONCLUSIONS}

Crenellated membrane of filled silicone were molded for different orientations of crenels. According to the literature, and previous work, an existing model to describe Mullins effect anisotropy is used. This model has been adapted to the crenel and a coupling term has been developed. Thus, every parts of the crenellated membrane can be evaluated. Several experimental tests were realized to validate the model. It has been observed that the coupling term could not be neglected on cyclic tensile test for different orientations of the crenels.

\section{REFERENCES}

Ashby, M. (2013). Designing architectured materials. Scripta Mater, 68, 4 - 7 .

Bazant, Z. P. and Oh, B. H. (1986). Efficient numerical integration on the surface of a sphere. Z. Angew. Math. Mech., 66, 37-49. 

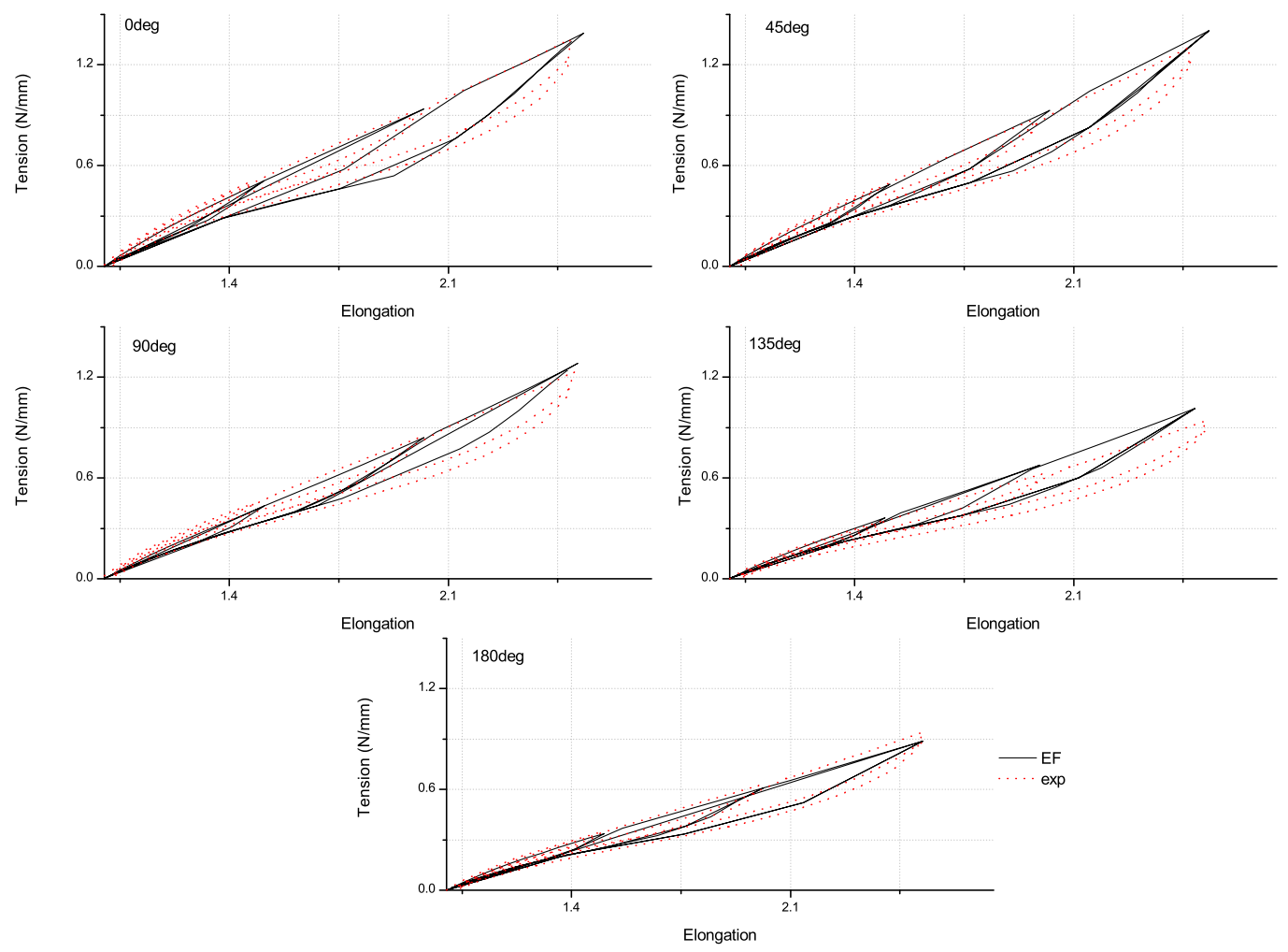

Figure 4: Comparison of experimental tests (dotted lines) and FE simulation (full lines) of architectured membrane for different orientations of the crenels with coupling

Bouaziz, O. (2013). Geometrically induced strain hardening. scripta mater, 68, $28-30$.

Bouaziz, O., Brechet, Y., and Embury, J. D. (2008). Heterogeneous and architectured materials: A possible strategy for design of structural materials. Adv. Engrg. Mater., 10, 24-36.

Brechet, Y. and Embury, J. (2013). Architectured materials: Expanding materials space. Scripta Mater, 68, $1-3$.

Dunlop, J. W. and Fratzl, P. (2013). Multilevel architectures in natural materials. Scripta Materialia, 68, $8-12$.

Göktepe, S., S. N. S. Acharya, J. W., and Kuhl, E. (2010). Computational modeling of passive myocardium. Int. J. Numer. Meth. Biomed. Engng., 27, 1-12.

Machado, G., Chagnon, G., and Favier, D. (2010). Analysis of the isotropic models of the Mullins effect based on filled silicone rubber experimental results. Mech. Mater., 42, 841-851.

Machado, G., Favier, D., and Chagnon, G. (2012a). Determination of membrane stress-strain full fields of bulge tests from SDIC measurements. Theory, validation and experimental results on a silicone elastomer. Exp. Mech., 52, 865-880.

Machado, G., Chagnon, G., and Favier, D. (2012b). Induced anisotropy by the mullins effect in filled silicone rubber. Mech. Mater, 50, $70-80$.

Meunier, L., Orgas, L., Chagnon, G., et Favier, D. (2009). Multiscale approach to model the mechanical behaviour of heterogeneous hyperelastic membranes. Constitutive Models for Rubber VI A. A. Balkema, 429-434.

Meunier, L. (2011). Contribution la conception, l'experimentation et la modélisation de membranes hyperélastiques architécturées anisotropes. Ph.D. thesis, Universite de Grenoble.

Mooney, M. (1940). A theory of large elastic deformation. J. Appl. Phys., 11, 582-592.

Natali, A. N., Carniel, E. L., and Gregersen, H. (2009). Biomechanical behaviour of oesophageal tissues: Material and structural configuration, experimental data and constitutive analysis. Med. Eng. \& Phys., 31, 1056-1062. 
Nerurkar, N. L., Mauck, R. L., and Elliott, D. M. (2011). Modeling interlamellar interactions in angle-ply biologic laminates for annulus fibrosus tissue engineering. Biomech Model Mechanobiol, 10, 973-984.

Peña, E. and Doblaré, M. (2009). An anisotropic pseudo-elastic approach for modelling Mullins effect in fibrous biological materials. Mech. Res. Comm., 36, 784-790.

Rebouah, M., Machado, G., Chagnon, G., and Favier, D. (2013). Anisotropic mullins stress softening of a deformed silicone holey plate. Mech. Res. Com., 49, 36-43.

Reese, S., Raible, T., and Wriggers, P. (2001). Finite element modelling of orthotropic material behaviour in pneumatic membranes. Int. J. Solids Struct., 38, 9525-9544.

Rey, T., Chagnon, G., Le Cam, J.-B., and Favier, D. (2013). Influence of the temperature on the mechanical behavior of unfilled and filled silicone rubbers above crystallization temperature. Polym. Test., 32, 492-501.

Spencer, A. J. M. (1971). Theory of Invariants. Continuum Physics, C. Eringen Academic Press. 